\section{Discontinuation of therapy in men with idiopathic hypogonadotropic hypogonadism}

A congenital defect in the release or action of gonadotropin-releasing hormone $(\mathrm{GnRH})$ can cause idiopathic hypogonadotropic hypogonadism, a condition that results in male infertility. Patients with this condition have incomplete or absent sexual maturation by the age of 18 years and were previously thought to require life-long treatment. Raivio and colleagues carried out a study to investigate whether discontinuation of hormonal therapy could cause neuroendocrine and gonadal reversal of idiopathic hypergonadotropic hypogonadism.

Patients with a diagnosis of idiopathic hypogonadotropic hypogonadism were eligible for inclusion. Following discontinuation of treatment, reversal was defined as a normal adult endogenous serum testosterone level of $\geq 270 \mathrm{ng} / \mathrm{dl}$.

Out of 50 men with idiopathic hypogonadotropic hypogonadism, 5 patients met the criteria for reversal of hypogonadism prospectively a mean $( \pm S D)$ of $6 \pm 3$ weeks after treatment discontinuation, and 10 patients were identified retrospectively. Of the 15 men, 6 had absent puberty and 9 had partial puberty at first presentation. All had abnormal secretion of $\mathrm{GnRH}$-induced luteinizing hormone and had received hormonal treatment to encourage fertility. Mean levels of endogenous testosterone, luteinizing hormone, follicle-stimulating hormone and testicular volume increased in patients who experienced reversal of hypogonadism.

This study reports a reasonably high incidence of sustained reversal. Patients with idiopathic hypogonadotropic hypogonadism should be informed of the possibility of spontaneous reversal of hypogonadism and fertility after discontinuation of reproductive hormonal therapy.

Original article Raivio T et al. (2007) Reversal of idiopathic hypogonadotropic hypogonadism. N Engl J Med 357: 863-873

\section{A novel regimen for poor-prognosis or relapsed germ cell tumors}

The optimum treatment regimen for patients with untreated poor-prognosis or relapsed germ cell tumors (GCT) is currently a matter of some debate. In a recent paper, researchers from St Bartholomew's Hospital, London, UK, describe a novel intensive protocol that is highly active in GCT.

Between September 1997 and June 2005, 27 patients with untreated poor-prognosis GCT, and 35 patients with relapsed GCT following conventional platinum-based therapy, were enrolled in a phase II trial of a novel intensive regimen. The cisplatin-based protocol-GAMEC-incorporated dactinomycin, etoposide and high-dose methotrexate, administered every 14 days. Following GAMEC administration and appropriate surgery, $74 \%$ of the previously untreated group and $51 \%$ of the previously treated group were progression-free at a median follow-up of 2.5 years. Furthermore, one patient in the previously untreated group and four patients in the pretreated group were salvaged by additional therapy. Unfortunately, the toxicity associated with the GAMEC regimen was considerable, with many cycles of therapy being complicated by cases of febrile neutropenia, approximately half of which required platelet transfusion. Overall, there were five treatment-related deaths - four owing to sepsis and one to intra-abdominal hemorrhage from choriocarcinoma. In the pretreated group, methotrexate dose density was significantly associated with progression-free survival $(P=0.003)$. Notably, neither the Memorial Sloan-Kettering Cancer Center criteria nor the Medical Research Council criteria for relapsed GCT were able to identify a poor-prognostic group for GAMEC.

The authors conclude that GAMEC is an effective treatment for patients with untreated or relapsed GCT, although it results in substantial toxic effects.

Original article Shamash J et al. (2007) GAMEC—a new intensive protocol for untreated poor prognosis and relapsed or refractory germ cell tumours. Br J Cancer 97: 308-314 